\title{
Integrable model of topological SO(5) superfluidity
}

\author{
Will J. Holdhusen $\odot,{ }^{1}$ Sergio Lerma-Hernández $\odot,{ }^{2}$ Jorge Dukelsky $\odot,{ }^{3}$ and Gerardo Ortiz $\oplus^{1}$ \\ ${ }^{1}$ Department of Physics, Indiana University, Bloomington, Indiana 47405, USA \\ ${ }^{2}$ Facultad de Física, Universidad Veracruzana, Circuito Aguirre Beltrán s/n, Xalapa, Veracruz 91000, Mexico \\ ${ }^{3}$ Instituto de Estructura de la Materia, CSIC, Serrano 123, 28006 Madrid, Spain
}

(Received 5 February 2021; revised 14 June 2021; accepted 26 July 2021; published 16 August 2021)

\begin{abstract}
Assisted by general symmetry arguments and a many-body invariant, we introduce a phase of matter that constitutes a topological SO(5) superfluid. Key to this finding is the realization of an exactly solvable model that displays some similarities with a minimal model of superfluid ${ }^{3} \mathrm{He}$. We study its quantum phase diagram and correlations, and find exotic superfluid as well as metallic phases in the repulsive sector. At the critical point separating trivial and nontrivial superfluid phases, our Hamiltonian reduces to the globally $\mathrm{SO}(5)$-symmetric Gaudin model with a degenerate ground manifold that includes quartet states. Most importantly, the exact solution permits uncovering of an interesting non-pair-breaking mechanism for superfluids subject to external magnetic fields. Nonintegrable modifications of our model lead to a strong-coupling limit of our metallic phase with a ground-state manifold that shows an extensive entropy.
\end{abstract}

DOI: 10.1103/PhysRevB.104.L060503

Introduction. Exactly solvable models of quantum manybody systems are theoretical constructions key to uncover physical mechanisms or effects resulting from competing interactions [1-3]. The case of spin- $1 / 2$ particles with $\mathrm{SO}(5)$ symmetric $p$-wave interactions is particularly compelling because it can give rise to nontrivial spin-triplet Cooper-pair topological phases with no equivalent in $\mathrm{SU}(2)$-symmetric couplings. For instance, it is well known that the emergent $\mathrm{SO}(3)_{\mathbf{L}} \otimes \mathrm{SO}(3)_{\mathbf{S}} \otimes \mathrm{U}(1)$ symmetry in liquid ${ }^{3} \mathrm{He}$, contained in $\mathrm{SO}(5)$, is responsible for topological classification of the defects of its exotic superfluid phases [4]. Similar mechanisms could be at play in unconventional uranium-based metallic ferromagnetic superconductors, where strong external magnetic fields can even revive superconductivity [5]. A theoretical understanding of these mechanisms is therefore a prerequisite to engineering materials or synthetic matter with exotic magnetic superfluid behavior [6].

$\mathrm{SO}(5)$-symmetric models have a long history in nuclear physics as a description of isovector (isospin 1) pairing between protons and neutrons. The earliest version of an integrable model consisting of a unique $\mathrm{SO}(5)$ algebra, describing a proton-neutron system, was presented in Refs. [7,8]. The generalization of the exact solution to many $\mathrm{SO}(5)$ copies or, equivalently, to many nondegenerate single-particle orbitals, arose as an extension of the Richardson-Gaudin (RG) models $[9,10]$ to rank 2 algebras [11,12]. In condensed matter physics, the systems closest to admitting an $\mathrm{SO}(5)$-symmetric representation are arguably superfluid ${ }^{3} \mathrm{He}$ [13-16] and non- $p$-wave systems [17-19], but, as far as we know, there are no corresponding integrable interacting models.

In this work, we study the quantum phase diagram of the fermionic $\left(c_{\mathbf{k} \sigma}, c_{\mathbf{k} \sigma}^{\dagger}\right) \mathrm{SO}(5)$ Hamiltonian expressed in momentum $(\mathbf{k})$ space as

$$
\begin{aligned}
H= & \sum_{\mathbf{k}, \sigma=\uparrow, \downarrow} \epsilon_{\mathbf{k}} c_{\mathbf{k} \sigma}^{\dagger} c_{\mathbf{k} \sigma}-\sum_{\mathbf{k}, \mathbf{k}^{\prime}} \Delta_{\mathbf{k} \mathbf{k}^{\prime}}\left(\vec{T}_{\mathbf{k}}^{+} \cdot \vec{T}_{\mathbf{k}^{\prime}}^{-}+\vec{T}_{\mathbf{k}}^{-} \cdot \vec{T}_{\mathbf{k}^{\prime}}^{+}\right) \\
& -\sum_{\mathbf{k}, \mathbf{k}^{\prime}} W_{\mathbf{k} \mathbf{k}^{\prime}} \vec{S}_{\mathbf{k}} \cdot \vec{S}_{\mathbf{k}^{\prime}}-\sum_{\mathbf{k}, \mathbf{k}^{\prime}} V_{\mathbf{k} \mathbf{k}^{\prime}} N_{\mathbf{k}} N_{\mathbf{k}^{\prime}}-h \sum_{\mathbf{k}} S_{\mathbf{k}}^{z} .
\end{aligned}
$$

The operator $T_{\mu \mathbf{k}}^{+}\left[\vec{T}_{\mathbf{k}}^{+}=\left(T_{-1 \mathbf{k}}^{+}, T_{0 \mathbf{k}}^{+}, T_{1 \mathbf{k}}^{+}\right)\right]$creates a spin-triplet fermion pair $(\mathbf{k},-\mathbf{k})$ with spin projection $\mu= \pm 1,0$. Magnetic Heisenberg $\left(\vec{S}_{\mathbf{k}} \cdot \vec{S}_{\mathbf{k}^{\prime}}\right)$ and density-density $\left(N_{\mathbf{k}} N_{\mathbf{k}^{\prime}}\right.$, where $N_{\mathbf{k}}$ counts all spinful fermions with momenta $\pm \mathbf{k}$ ) interactions complete the minimal set required to close an $\mathrm{SO}(5)$ algebra, with a fermionic representation [20]

$$
\begin{aligned}
T_{\mu \mathbf{k}}^{-} & =\frac{(-1)^{\frac{\mu(\mu+1)}{2}}}{\left(2 \delta_{\mu, \pm 1}+\sqrt{2} \delta_{\mu, 0}\right)} \sum_{\sigma, \sigma^{\prime}} c_{-\mathbf{k} \sigma}\left(i \sigma^{\mu} \sigma^{y}\right)_{\sigma \sigma^{\prime}} c_{\mathbf{k} \sigma^{\prime}}, \\
S_{\mathbf{k}}^{\mu} & =\frac{1}{2} \sum_{\sigma, \sigma^{\prime}}\left(c_{\mathbf{k} \sigma}^{\dagger} \sigma_{\sigma \sigma^{\prime}}^{\mu} c_{\mathbf{k} \sigma^{\prime}}+c_{-\mathbf{k} \sigma}^{\dagger} \sigma_{\sigma \sigma^{\prime}}^{\mu} c_{-\mathbf{k} \sigma^{\prime}}\right), \\
N_{\mathbf{k}} & =\sum_{\sigma}\left(c_{\mathbf{k} \sigma}^{\dagger} c_{\mathbf{k} \sigma}+c_{-\mathbf{k} \sigma}^{\dagger} c_{-\mathbf{k} \sigma}\right),
\end{aligned}
$$

where $\sigma^{ \pm}=\sigma^{x} \pm i \sigma^{y}, \sigma^{0}=\sigma^{z}$ are Pauli matrices, and $T_{\mu \mathbf{k}}^{+}=$ $\left(T_{\mu \mathbf{k}}^{-}\right)^{\dagger}$. For an appropriate choice of (separable) interactions $\Delta_{\mathbf{k} \mathbf{k}^{\prime}}=W_{\mathbf{k k}^{\prime}}=4 V_{\mathbf{k k}^{\prime}}$, Hamiltonian (1) is exactly solvable independently of spatial dimensionality.

In the attractive pairing sector, the model displays trivial and nontrivial topological superfluid phases, separated by a critical point that is globally $\mathrm{SO}(5)$-symmetric. At this point the ground-state manifold is macroscopically degenerate with pair and quartet correlations. The application of a magnetic field $h$ [21] leads to a remarkable magnetized superfluid, 
where spin-triplet pairs transition, without pair breaking, between different spin projections as in the B to A first-order transition in superfluid ${ }^{3} \mathrm{He}$. This mechanism is absent in $\mathrm{SU}(2)$ pairing models. Finally, the repulsive sector shows a metallic phase whose strong-coupling limit is adiabatically connected to a flat-band model with exponentially degenerate ground states, giving an extensive entropy similar to holographic models of strange metals [22].

Exactly solvable SO(5) model. RG integrable models are defined by a set of integrals of motion $R_{\mathrm{k}}$ fulfilling the integrability condition $\left[R_{\mathrm{k}}, R_{\mathbf{k}^{\prime}}\right]=0$, such that their linear combination realizes a Hamiltonian as (10). The exact eigenspectrum of the integrals of motion, and corresponding Hamiltonian, may be found with algebraic complexity by solving the RG ansatz equations.

These models may be formulated in terms of a generalized Gaudin algebra [3,23-25]. Starting from the rational $\mathrm{SO}(5)$ RG integrals of motion [12], we form the set

$$
R_{\mathbf{k}}=\left(1+\frac{\Delta}{2}\right) N_{\mathbf{k}}^{-}+\frac{\Delta}{2} S_{\mathbf{k}}^{z}+q \sum_{\mathbf{k}^{\prime} \neq \mathbf{k}} Z_{\mathbf{k} \mathbf{k}^{\prime}} \overrightarrow{\mathcal{T}}_{\mathbf{k}} \cdot \overrightarrow{\mathcal{T}}_{\mathbf{k}^{\prime}},
$$

where $N_{\mathbf{k}}^{-}=N_{\mathbf{k}} / 2-1$, and $\overrightarrow{\mathcal{T}}_{\mathbf{k}} \cdot \overrightarrow{\mathcal{T}}_{\mathbf{k}^{\prime}}$ is the $\mathrm{SO}(5)$ Gaudin interaction $\overrightarrow{\mathcal{T}}_{\mathbf{k}} \cdot \overrightarrow{\mathcal{T}}_{\mathbf{k}^{\prime}}=\vec{T}_{\mathbf{k}}^{+} \cdot \vec{T}_{\mathbf{k}^{\prime}}^{-}+\vec{T}_{\mathbf{k}}^{-} \cdot \vec{T}_{\mathbf{k}^{\prime}}^{+}+\vec{S}_{\mathbf{k}} \cdot \vec{S}_{\mathbf{k}^{\prime}}+N_{\mathbf{k}}^{-} N_{\mathbf{k}^{\prime}}^{-}$ The function $Z_{\mathbf{k} \mathbf{k}^{\prime}}=Z\left(\eta_{\mathbf{k}}, \eta_{\mathbf{k}^{\prime}}\right)=\frac{\eta_{\mathbf{k}} \eta_{\mathbf{k}^{\prime}}}{\eta_{\mathbf{k}}-\eta_{\mathbf{k}^{\prime}}}, Z_{\mathbf{k}^{\prime}}=-Z_{\mathbf{k}^{\prime} \mathbf{k}}$, is a particular case of a more general function interpolating between hyperbolic and trigonometric $\mathrm{SU}(2) \mathrm{RG}$ models $[3,26,27]$. The parameters $\Delta, q$, and $\eta_{\mathbf{k}}$ are arbitrary real numbers with the restriction that $\eta_{\mathbf{k}} \neq \eta_{\mathbf{k}^{\prime}}$ for $\mathbf{k} \neq \mathbf{k}^{\prime}$ to avoid singularities.

In Eq. (3) and for the remainder of this Letter, sums are taken over momenta with $k_{x}>0$ to avoid double counting. Each pair of momenta $\pm \mathbf{k}$ labels a level with a corresponding irreducible representation (irrep) of $\mathrm{SO}(5)$ characterized by seniority $v_{\mathbf{k}}$ and reduced spin $s_{\mathbf{k}}$ quantum numbers. The $l$ levels correspond to a lattice with $L=2 l$ sites, since each level incorporates two modes in $\mathbf{k}$ space.

Eigenvalues and eigenvectors of the integrals of motion are determined by two sets of spectral parameters: pairons $e_{\alpha}, \alpha=$ $1, \ldots, N_{e}$, and wave function parameters $\omega_{\beta}, \beta=1, \ldots, N_{\omega}$, that are roots of the two sets of RG (Bethe) equations

$$
-\frac{1}{q}=2 \sum_{\alpha^{\prime} \neq \alpha} Z_{\alpha^{\prime} \alpha}-\sum_{\beta} Z_{\beta \alpha}+\sum_{\mathbf{k}}\left(\frac{\nu_{\mathbf{k}}}{2}-1+s_{\mathbf{k}}\right) Z_{\mathbf{k} \alpha}
$$

and

$$
-\frac{\Delta}{q}=-\sum_{\beta^{\prime} \neq \beta} Z_{\beta^{\prime} \beta}+\sum_{\alpha} Z_{\alpha \beta}+\sum_{\mathbf{k}} s_{\mathbf{k}} Z_{\mathbf{k} \beta},
$$

with $\quad Z_{\alpha^{\prime} \alpha}=Z\left(e_{\alpha^{\prime}}, e_{\alpha}\right), \quad Z_{\beta \alpha}=Z\left(\omega_{\beta}, e_{\alpha}\right), \quad$ and $\quad Z_{\mathbf{k} \alpha}=$ $Z\left(\eta_{\mathbf{k}}, e_{\alpha}\right)$. The number of pairons $N_{e}$ is equal to the number of spin-1 fermion pairs and relates to the total fermion number as $N=2 N_{e}+\sum_{\mathbf{k}} \nu_{\mathbf{k}}$. The number of wave function parameters is $N_{\omega}=N_{e}+\sum_{\mathbf{k}}\left(S_{\mathbf{k}}^{z}+s_{\mathbf{k}}\right)$. We emphasize that while the dimension of the Hilbert space grows exponentially with the number of levels $L$ and $N_{e}$, the complexity of the exact solution grows only polynomially, allowing exact treatment of very large systems.

Numerical solution of the RG equations must navigate the singularities that arise whenever the spectral parameters approach each other or the level parameters $\eta_{\mathbf{k}}$. To avoid these singularities, we add modulated imaginary parts to $\eta_{\mathbf{k}}$ while iteratively solving to a desired coupling $q$ and then incrementally remove these to achieve physical results [27].

In terms of the variables $e_{\alpha}$ and $\omega_{\beta}$, the integrals of motion $R_{\mathrm{k}}$ have eigenvalues

$$
\begin{aligned}
r_{\mathbf{k}}= & \frac{\nu_{\mathbf{k}}}{2}-1+q s_{\mathbf{k}} \sum_{\beta} Z_{\mathbf{k} \beta}+q\left(1-\frac{\nu_{\mathbf{k}}}{2}-s_{\mathbf{k}}\right) \sum_{\alpha} Z_{\mathbf{k} \alpha} \\
& -q \sum_{\mathbf{k}^{\prime} \neq \mathbf{k}}\left[\left(\frac{\nu_{\mathbf{k}}}{2}-1\right)\left(\frac{\nu_{\mathbf{k}^{\prime}}}{2}-1\right)+s_{\mathbf{k}} s_{\mathbf{k}^{\prime}}\right] Z_{\mathbf{k} \mathbf{k}^{\prime}} .
\end{aligned}
$$

To obtain the corresponding eigenstates, we need operators

$$
\mathbf{S}_{\beta}^{+}=\sum_{\mathbf{k}} Z_{\mathbf{k} \beta} S_{\mathbf{k}}^{+}, \quad \mathbf{T}_{\mu \alpha}^{+}=\sum_{\mathbf{k}} Z_{\mathbf{k} \alpha} T_{\mu \mathbf{k}}^{+},
$$

and $\overleftarrow{\mathrm{I}}_{\alpha}^{+}$, defined by its action on $\mathrm{T}_{\mu \alpha}^{+}$:

$$
\mathrm{T}_{\mu \alpha}^{+} \overleftarrow{\mathrm{T}}_{\alpha^{\prime}}^{+}=\delta_{\alpha \alpha^{\prime}} \begin{cases}\mathrm{T}_{\mu+1 \alpha}^{+}, & \mu \leqslant 0 \\ 0, & \mu=1\end{cases}
$$

Then, the eigenstates can be written as [20]

$$
|\Psi\rangle=\prod_{\alpha=1}^{N_{e}} \mathrm{~T}_{-1 \alpha}^{+} \prod_{\beta=1}^{N_{\omega}}\left(\mathrm{S}_{\beta}^{+}-\sum_{\alpha^{\prime}=1}^{N_{e}} \overleftarrow{\mathrm{T}}_{\alpha^{\prime}}^{+} Z_{\alpha^{\prime} \beta}^{*}\right)|\Lambda\rangle,
$$

where $|\Lambda\rangle$ is a vacuum state characterized by $\nu_{\mathbf{k}}$ and $s_{\mathbf{k}}$ with $S_{\mathbf{k}}^{-}|\Lambda\rangle=T_{\mu \mathbf{k}}^{-}|\Lambda\rangle=0$. In most cases, the ground state is built from the empty ( $v_{\mathbf{k}}=s_{\mathbf{k}}=0$ for all $\mathbf{k}$ ) vacuum $|0\rangle$. The exception occurs when sufficiently strong repulsive pairing couplings break pairs.

When $\epsilon_{\mathbf{k}}=\eta_{\mathbf{k}}, \quad \Delta_{\mathbf{k k}^{\prime}}=W_{\mathbf{k} \mathbf{k}^{\prime}}=4 V_{\mathbf{k k}^{\prime}}=(g / L) \eta_{\mathbf{k}} \eta_{\mathbf{k}^{\prime}}$, and $h=0$, Hamiltonian (1) can be written as a linear combination of the integrals of motion [20]

$$
\begin{aligned}
H & =\frac{2}{1-q \sum_{\mathbf{k}} \eta_{\mathbf{k}}} \sum_{\mathbf{k}} \eta_{\mathbf{k}} R_{\mathbf{k}}+\text { constant } \\
& =\left(1-\frac{g}{g_{c}}\right) \sum_{\mathbf{k}} \eta_{\mathbf{k}} N_{\mathbf{k}}-\frac{g}{L} \sum_{\mathbf{k}, \mathbf{k}^{\prime}} \eta_{\mathbf{k}} \eta_{\mathbf{k}^{\prime}} \overrightarrow{\mathcal{T}}_{\mathbf{k}} \cdot \overrightarrow{\mathcal{T}}_{\mathbf{k}^{\prime}}+\frac{g L}{g_{c}^{2}} .
\end{aligned}
$$

Here, we define $q L=-g /\left(1-g / g_{c}\right)$ with $g_{c}^{-1} L=\sum_{\mathbf{k}} \eta_{\mathbf{k}}$ and set $\Delta=0$ (letting $\Delta \neq 0$ has the effect of assigning a different kinetic energy to spin-up versus spin-down fermions). At $g=$ $g_{c}, q$ becomes singular and $H$ reduces to the (globally) $\mathrm{SO}(5)-$ symmetric Gaudin model, as is evident from the second line in Eq. (10). Adding a uniform magnetic field $h$ does not break integrability and is discussed below.

Using the eigenvalues $r_{\mathbf{k}}$ from Eq. (6), the total energy for a system of density $\rho=N / L$ is

$$
\mathcal{E}(N)=\frac{2 \sum_{\mathbf{k}} \eta_{\mathbf{k}} r_{\mathbf{k}}}{1-q \sum_{\mathbf{k}} \eta_{\mathbf{k}}}
$$

up to a constant dependent on the vacuum state $|v\rangle$ [20] while total energy per site (energy density) will be indicated by $e=\mathcal{E}(N) / L$. Other observables may also be computed from the integrals of motion using the Hellmann-Feynman theorem, e.g., momentum distribution $\left\langle N_{\mathbf{k}}\right\rangle=2\left(r_{\mathbf{k}}-q \partial r_{\mathbf{k}} / \partial q+1\right)$.

Hamiltonian (10) displays a particle-hole symmetry $\mathcal{P}$. Under the map $\mathcal{P}^{\dagger} c_{\mathbf{k} \uparrow} \mathcal{P}=c_{\mathbf{k} \downarrow}^{\dagger}, \mathcal{P}^{\dagger} c_{\mathbf{k} \downarrow} \mathcal{P}=c_{\mathbf{k} \uparrow}^{\dagger}, H$ transforms 


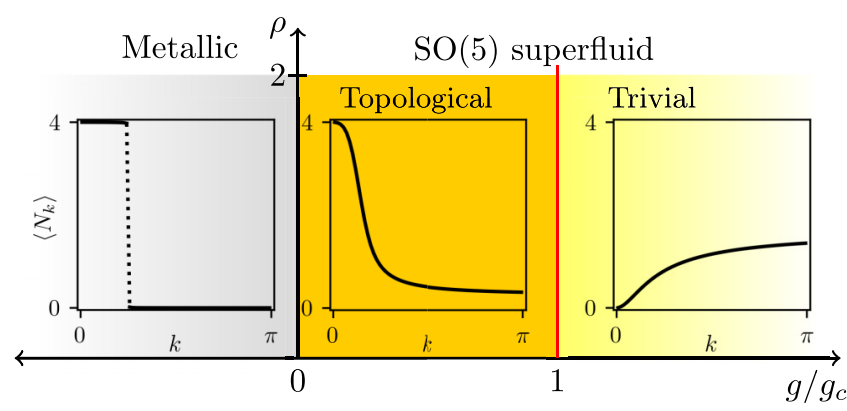

FIG. 1. Quantum phase diagram of the SO(5) RG model as a function of density $\rho$ and coupling $g$. Ground-state momentum distributions $\left\langle N_{k}\right\rangle$, as a function of $k>0$ for $\rho=1 / 2$, are displayed at $g / g_{c}=-1, \frac{3}{4}, \frac{3}{2}$, that is, for the metallic, topological, and trivial paired-superfluid phases, respectively. The metallic phase displays a discontinuity at the Fermi momentum $k_{F}=\pi \rho / 2$, the topological superfluid phase is continuous with occupation of low-momentum modes, and the trivial superfluid vacates the low-momentum modes. The phase transition between superfluid phases is second order (see Fig. 2).

as $\mathcal{P}^{\dagger} H(\rho, g) \mathcal{P}=\alpha H(2-\rho, \alpha g)$ up to an additive constant, where $\alpha^{-1}=2 g / g_{c}-1$. At $g=g_{c}, \alpha=1$ and $H$ is particlehole symmetric.

Quantum phase diagram: One-dimensional case. To illustrate the physics arising from our $\mathrm{SO}(5)$ model we now work in one spatial dimension. The momenta for periodic boundary conditions are $k_{j}=\frac{2 \pi j}{L}, j=-L / 2,-L / 2+1, \ldots, L / 2-1$, which leaves isolated modes at $k=0$ and $k=-\pi$ that cannot participate in pairing interactions and therefore do not correspond to RG levels. When using these boundary conditions, we ignore all interactions on the $k=-\pi$ mode to preserve integrability. The effect of the ignored interactions diminishes in the thermodynamic limit $(L \rightarrow \infty$ with $\rho$ and $g$ fixed). To avoid this finite-size effect, the majority of our calculations utilize antiperiodic boundary conditions, under which all momenta come in pairs $(+|k|,-|k|)$ corresponding to RG levels: $k_{j}=\frac{\pi}{L}(2 j+1), j=-L / 2,-L / 2+1, \ldots, L / 2-1$.

We linearize the dispersion close to the Fermi points by choosing $\eta_{k}=k$ and $\epsilon_{k}=|k|$ (in units of $\frac{1}{2} \hbar v_{F}$ where $\hbar$ is the reduced Planck's constant and $v_{F}$ the Fermi velocity). Because $\eta_{k}=-\eta_{-k}$, interaction coefficients $\Delta_{k k^{\prime}}=$ $\eta_{k} \eta_{k^{\prime}}$ have the antisymmetry necessary for $p$-wave pairing: $\Delta_{k k^{\prime}}=\Delta_{-k-k^{\prime}}=-\Delta_{-k k^{\prime}}$. In coordinate space, the Fouriertransformed coefficients $\Delta_{i j}$ linking sites at $r_{i}$ and $r_{j}$ decay as $\left(r_{i}-r_{j}\right)^{-1}$ [28,29], and show alternating sign $(-1)^{i-j}$ [20].

We next analyze the various phases that emerge in the phase diagram of the SO(5) RG Hamiltonian (10).

Topological superfluid phase. For attractive couplings $g>$ 0 , the ground state of Hamiltonian (10) is a superfluid of spin-triplet pairs. At the density-independent critical coupling $g=g_{c}$, the system undergoes a topological phase transition with an accompanying change in occupation number $\left\langle N_{k}\right\rangle$ around zero momentum $(k=0)$, as seen in the inset of Fig. 1 . The system transitions from a weak-pairing topologically nontrivial $\mathrm{SO}(5)$ superfluid into a strong-pairing trivial superfluid gapped phase. The transition is signaled by a divergence in $\partial^{2} e_{0} / \partial g^{2}$, the second-order derivative of the ground-state

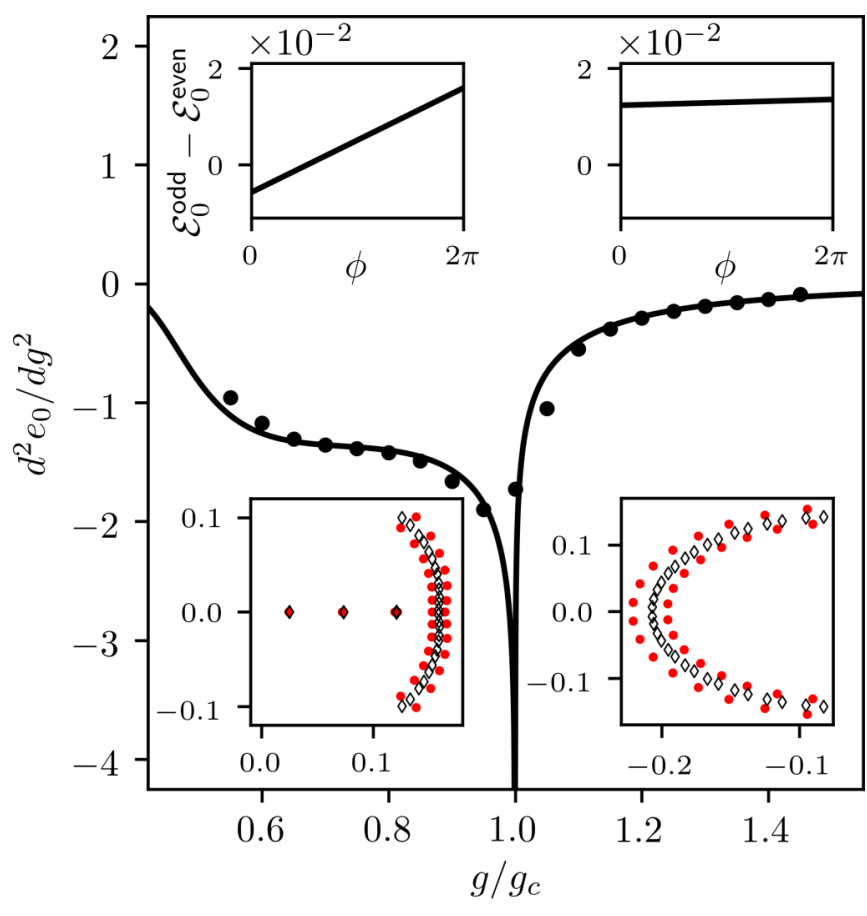

FIG. 2. Second derivative of the ground-state energy density $e_{0}$ with respect to coupling for a mean-field solution [20] with $L=2000$ (solid line), and the exact solution at $L=128$, both at quarter filling ( $\rho=1 / 2$ ). Lower insets: The variables $e_{\alpha}$ (diamonds) and $\omega_{\beta}$ (dots) are plotted with their imaginary parts on the $y$ axis and their real parts on the $x$ axis at $g / g_{c}=0.9$ (left) and 1.1 (right). An animation of these spectral parameters as a function of $g$ is included in the Supplemental Material [20]. Upper insets: Energy difference between odd and even sectors is plotted as a function of boundary condition $\phi$ for a quarter-filled system with 32 fermions at $g / g_{c}=0.7$ (left) and 1.75 (right).

energy density. Figure 2 illustrates this along with distribution of spectral parameters in the complex plane.

To understand the topological nature of these superfluid phases, we need a many-body (bulk) topological invariant distinguishing them. Reference [28] introduces a fermion parity switch for spinless fermions that distinguished $p$-wave topological phases of an $\mathrm{SU}(2)$ model. Our $\mathrm{SO}(5)$ model consists of spinful fermions and therefore requires a generalization of the fermion parity to

$$
\mathcal{P}_{N}(\phi)=\operatorname{sgn}\left(\mathcal{E}_{0}^{\text {odd }}(\phi)-\mathcal{E}_{0}^{\text {even }}(\phi)\right),
$$

where the ground-state energies are defined as $\mathcal{E}_{0}^{\text {even }}(\phi)=$ $\mathcal{E}_{0}^{\phi}(N)$ and $\mathcal{E}_{0}^{\text {odd }}(\phi)=\frac{1}{2}\left[\mathcal{E}_{0}^{\phi}(N+2)+\mathcal{E}_{0}^{\phi}(N-2)\right]$ for fermion number $N$ divisible by four, such that the $N \pm 2$-particle states have $N_{\uparrow}=N_{\downarrow}$ odd. This differs from the $\mathrm{SU}(2)$ case (where $\mathcal{E}_{0}^{\text {odd }}$ is the average of $N \pm 1$-particle energies) due to the spin degeneracy of the $k=0$ mode. The quantity $\phi=0(2 \pi)$ represents periodic (antiperiodic) boundary conditions and corresponds to enclosing a flux $\Phi=\frac{\phi \Phi_{0}}{2 \pi}$ in a ring geometry with anomalous flux quantum $\Phi_{0}$ [28,29]. In the topologically trivial phase $\left(g>g_{c}\right)$, $\mathcal{P}_{N}(\phi)=1$ for both periodic and antiperiodic boundary conditions (Fig. 2). For $g<g_{c}$, a parity switch is observed, with $\mathcal{P}_{N}(0)=-1$ and $\mathcal{P}_{N}(2 \pi)=1$. This can be linked back 
to occupation of the zero-momentum state that exists for $\phi=0$ : in the topologically nontrivial phase, it is energetically advantageous to occupy both $(\sigma=\uparrow, \downarrow) k=0$ single-particle states instead of forming a pair. In the pairing-dominated trivial phase, the $k=0$ states are vacated in favor of an additional pair at finite momentum.

Unlike what is seen in other SU(2) RG models, there is a macroscopic degeneracy at the critical point involving multiple states from each sector with fixed $N$ and $M=\sum_{\mathbf{k}} S_{\mathbf{k}}^{z}$, with an accompanying global $\mathrm{SO}(5)$ symmetry generated by operators $I^{\kappa}=\sum_{\mathbf{k}} I_{\mathbf{k}}^{\kappa}$ where $I_{\mathbf{k}}^{\kappa}, \kappa=1, \ldots, 10$, is any generator of the $\mathrm{SO}(5)$ algebra in Eq. (2). At $g=g_{c}$, Hamiltonian (10) becomes the $\mathrm{SO}(5)$ Gaudin model $\sum_{\mathbf{k}, \mathbf{k}^{\prime}} \eta_{\mathbf{k}} \eta_{\mathbf{k}^{\prime}} \overrightarrow{\mathcal{T}}_{\mathbf{k}} \cdot \overrightarrow{\mathcal{T}}_{\mathbf{k}^{\prime}}$, and the ground-state solutions to the $\mathrm{RG}$ equations have all pairons $e_{\alpha}$ equal to zero. Those equations then simplify to a single set for variables $\omega_{\beta}, \sum_{\beta^{\prime} \neq \beta} Z_{\beta^{\prime} \beta}=\sum_{\mathbf{k}} s_{\mathbf{k}} Z_{\mathbf{k} \beta}, \beta=$ $1, \ldots, N_{\omega}$. Each independent solution corresponds to a degenerate eigenstate. The entire energy spectrum at this point can be classified according to the degenerate $\mathrm{SO}(5)$ global irreps constructed from the coupling of the $l \mathrm{SO}(5)_{\mathbf{k}}$ irreps $\left\{v_{\mathbf{k}}, s_{\mathbf{k}}\right\}$ of each level. The chain decomposition $\mathrm{SO}(5) \supset$ $\mathrm{U}_{\mathbf{S}}(2) \supset \mathrm{U}_{S^{z}}(1)$ [30] classifies the complete set of eigenstates in terms of the fermion number $N$ and spin content $S$ in each global irrep. The wave functions constituting the groundstate irrep are defined in terms of $S=0$ quartet creation operator, $Q^{+}=\sum_{\mathbf{k}, \mathbf{k}^{\prime}}\left(T_{1 \mathbf{k}}^{+} T_{-1 \mathbf{k}^{\prime}}^{+}+T_{-1 \mathbf{k}}^{+} T_{1 \mathbf{k}^{\prime}}^{+}-T_{0 \mathbf{k}}^{+} T_{0 \mathbf{k}^{\prime}}^{+}\right), S=1$ global pair operators $T_{\mu}^{+}=\sum_{\mathbf{k}} T_{\mu \mathbf{k}}^{+}$, and spin-lowering operator $S^{-}=\sum_{\mathbf{k}} S_{\mathbf{k}}^{-}$. For an even number of particles $N \leqslant L$ ( $N>L$ states can be determined by particle-hole transformation), these states are

$$
\left|N_{Q}, S, M\right\rangle=\left(S^{-}\right)^{S-M}\left(Q^{+}\right)^{N_{Q}}\left(T_{1}^{+}\right)^{S}|0\rangle .
$$

Since $N=4 N_{Q}+2 S$, the possible values of spin are $S=$ $N / 2, N / 2-2, \ldots, 1$ or 0 , with $S=0$ representing the pure quartet state. From this, we find the degeneracy of the even- $N$ ground-state manifold: $d_{N, M}^{\text {even }}=\left\lfloor\frac{\min (N, 2 L-N)-2|M|}{4}\right\rfloor+1$, where $\lfloor x\rfloor$ is the largest integer less than or equal to $x$. The energy of these states is $\mathcal{E}^{\text {even }}=-3 \frac{g_{c}}{L} \sum_{\mathbf{k}} \eta_{\mathbf{k}}^{2}$.

The $N+1$ ( $N$ even) particle ground-state irrep has $N$ particles in a wave function of the form (13) with spin $S_{e}$ and one unpaired particle in the lowest momentum level $k_{m}$, giving total spin $S=S_{e} \pm 1 / 2$ with possible values $S=N / 2, N / 2-$ $1, \ldots, 1 / 2$. The number of particles is then $N=4 N_{Q}+2 S_{e}+$ 1 . From the available spins and the additional twofold degeneracy arising from the two momenta $\left( \pm \mathbf{k}_{m}\right)$ of the unpaired particle, the degeneracy of the odd-sector ground-state subspace is $d_{N, M}^{\text {odd }}=\frac{\min (N, 2 L-N)-2|M|}{2}+1$. The energy of these states $\mathcal{E}^{\text {odd }}=\mathcal{E}^{\text {even }}+\eta_{k_{m}}\left(1+\frac{g_{c}}{2 L} \eta_{k_{m}}\right)$ simplifies to the even$N$ energy plus the kinetic energy of the unpaired fermion in the thermodynamic limit.

The presence of quartets in a Hamiltonian such as (10) deserves mention. We are only aware of the significance of quartet correlations in atomic nuclei $[31,32]$ and in exotic phases of cold spin-3/2 fermionic atoms [17,19]. It is important, then, to establish the interactions that take our system away from its $g=g_{c}$ critical point and stabilize a quartet, as opposed to a paired, ground state. To this end, we compare the quartet, $\Delta_{4}(N)=\left[\mathcal{E}_{0}(N+2)+\mathcal{E}_{0}(N-2)-2 \mathcal{E}_{0}(N)\right] / 2$ (see for example [33]), and paired, $\Delta_{2}(N)=\left[\mathcal{E}_{0}(N+1)+\right.$
$\left.\mathcal{E}_{0}(N-1)-2 \mathcal{E}_{0}(N)\right] / 2$, gaps in our Hamiltonian (10) as a function of $g$. If $\Delta_{4}(N) \sim \Delta_{2}(N)$, we say that there are significant quartet correlations in the ground state for that value of $g$. Our analysis indicates that quartet correlations become more relevant in the repulsive sector and, in the attractive sector, for pairing-only (nonintegrable) interactions [20].

$S O(5)$ magnetic superfluid. An interesting physical mechanism emerges when our $\mathrm{SO}(5)$ system (10) is subject to an external magnetic field $h$ as in Eq. (1). At low temperatures and pressures superfluid ${ }^{3} \mathrm{He}$, known to have both $p$-wave pairing and ferromagnetic interactions [13], displays transitions between nonmagnetic (B) and magnetic (A) superfluid phases as function of an applied magnetic field. The A and B phases of superfluid ${ }^{3} \mathrm{He}$ are associated with the mean-field wave functions proposed by Anderson, Brinkman, and Morel $(\mathrm{ABM})$ and Balian and Werthamer (BW), respectively [13]. The BW state is a simple generalization of BCS principles to spin-triplet (rather than spin-singlet) pairs, and in the $\mathrm{SO}(5)$ language is a superposition of $T_{-1}^{+}, T_{0}^{+}$, and $T_{1}^{+}$operators acting on the vacuum. The ABM state is structured similarly, but allows only like-spin fermion pairs, ruling out the channel generated by $T_{0}^{+}$. Experimentally, it is known that in absence of a magnetic field, the B phase is the only possible superfluid at zero temperature. With the addition of a magnetic field, both phases become accessible at zero temperature along with the spin-polarized superfluid A1 phase [34].

Interestingly, our model demonstrates a series of first-order magnetically driven transitions between different spin-triplet superfluids with no pair breaking, which may provide insight into magnetic superfluidity. To determine the ground-state energy of the Hamiltonian (10), it suffices to find $\mathcal{E}_{0}(N, M)$, the lowest energy of the $h=0$ Hamiltonian for each possible value of $S^{z}$ (a conserved quantity), and determine which value of $M$ gives the lowest total energy $\mathcal{E}_{0}(h)=\min _{M}\left(\mathcal{E}_{0}(N, M)-\right.$ $h M)$. This process simplifies for $2 g>g_{c}$, since above this coupling we find the ground state has no unpaired fermions for any value of $h$. From this formula, it is clear that the magnetization $\mathcal{M}_{z}=\partial \mathcal{E}_{0} / \partial h$ is equal to $M$. Rather than breaking pairs, the magnetic field changes the balance of $-1,0$, and 1 pairs. This manifests as a series of first-order phase transitions as $\mathcal{M}_{z}$ jumps between integers with the same parity as $N_{e}$, as illustrated in Fig. 3. The minimum value of $h$ for nonzero magnetization goes to zero in the thermodynamic limit, while the final transition occurs at a value, $h=h_{c}$, that remains finite in that limit. A similar type of mechanism may be at play in superfluid ${ }^{3} \mathrm{He}$ leading to the emergence of the A1 phase. Crucially, the $h \neq 0$ ground state of our model is merely the lowest-energy solution to the $h=0$ problem at the same coupling $g$ in a sector with $N_{\uparrow} \neq N_{\downarrow}$, and so shares topological and superfluid properties with the $h=0$ ground state at the same coupling $g$.

This non-pair-breaking mechanism, already encoded in the exact solution, can be modeled at the mean-field level by introducing the $\mathrm{SO}(5)$ generalized coherent state [35]

$$
|\Psi\rangle=e^{\sum_{k} z_{k}\left(x_{1} T_{0 k}^{+}+x_{2} T_{1 k}^{+}+x_{3} T_{-1 k}^{+}\right)}|0\rangle
$$

where $x_{1}^{2}+x_{2}^{2}+x_{3}^{2}=1$ and $\left\{z_{k}\right\}$ are variational parameters [20]. As $\left|x_{1,2,3}\right|$ goes from 0 to 1 , this state goes from having only $M=0$ pairs through a state with a mixture of all three 


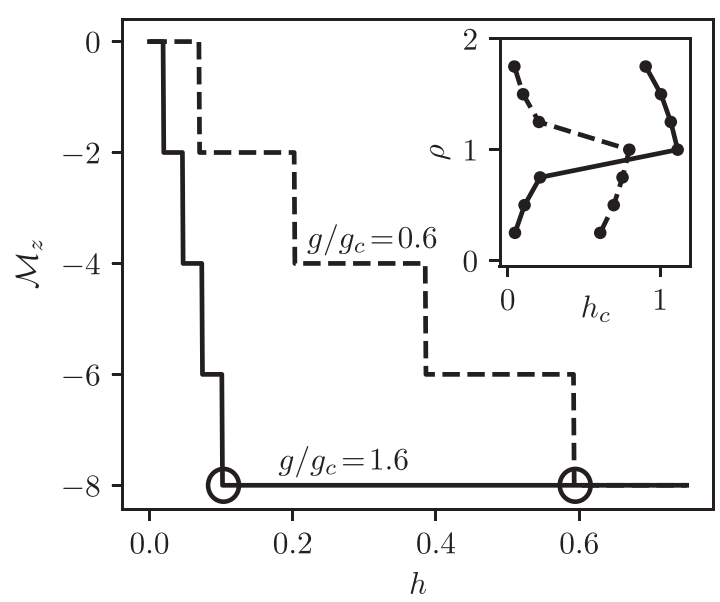

FIG. 3. Ground-state magnetization $\mathcal{M}_{z}$ as a function of $h$ at $g / g_{c}=0.6$ (dashed line) and 1.6 (solid line) for $L=32$ and $N=16$ $(\rho=1 / 2)$. The circled points are at $h=h_{c}$, the smallest field that fully polarizes the system. Inset: Density $\rho$ as a function of the thermodynamic extrapolation of $h_{c}$, indicating a phase boundary between fully and semipolarized magnetic superfluids.

pairing channels, similar to the BW state, to a state with only like-spin pairs, similar to the ABM wave function.

Metallic phases. For repulsive couplings $(g<0)$, the ground state has a momentum distribution with a discontinuity at the Fermi momentum $k_{F}$ (Fig. 1), suggesting a ground state almost identical to a noninteracting Fermi gas $\left|\Psi_{\text {nonint }}\right\rangle=\frac{1}{\sqrt{N !}} \prod_{k=-k_{F}}^{k_{F}} c_{k \uparrow}^{\dagger} c_{k \downarrow}^{\dagger}|0\rangle$ with energy $\mathcal{E}_{\text {nonint }}(N)=\left\langle\Psi_{\text {nonint }}|H| \Psi_{\text {nonint }}\right\rangle$. This discontinuity persists even for strongly repulsive couplings, unlike what is usually observed in an interacting Fermi liquid $[3,36]$. In the thermodynamic limit, the ground-state energy density $e_{0}$ converges to $e_{\text {nonint }}=\frac{\pi}{4} \rho^{2}-\frac{g \pi^{2}}{64} \rho^{4}+O(1 / L)[20]$.

One may wonder whether Eq. (10) has a flat-band limit in the strong-coupling $(g \rightarrow-\infty)$ limit. The SU(2) RG model shares similar metallic properties with the $\mathrm{SO}(5)$ model for low couplings, but the flat-band Hamiltonian $\lim _{g \rightarrow-\infty} \frac{1}{g} H$ has an exponentially degenerate ground-state manifold [20]. This limiting case has been studied, for instance, in fractional quantum Hall liquids [37], and its importance lies in the nonFermi-liquid behavior that manifests due to a high density of states near the ground state. Due to the presence of effective single-particle terms in the interaction, the $\mathrm{SO}(5)$ model in (10) does not exhibit high degeneracy in this limit. Instead, a level crossing occurs at a nonuniversal coupling where the ground state gains a nonzero seniority independent of system size [20]. By removing all single-particle terms, one arrives at a special case of the Hamiltonian (1) with an exponentially degenerate ground state in the flat-band (pure interaction) limit. A detailed discussion of this behavior is beyond the scope of this Letter [20].

Concluding remarks. We have presented an exactly solvable model displaying $\mathrm{SO}(5)$ topological superfluidity. Its relevance lies in providing a new non-pair-breaking mechanism for magnetic superfluids, of relevance for liquid ${ }^{3} \mathrm{He}$ or other exotic spin-triplet $p$-wave superfluids. At a critical coupling separating trivial and nontrivial topological superfluids, the model reduces to an (global) SO(5) Gaudin Hamiltonian. These phases show quartet correlations that become more prevalent as magnetic and density interactions are quenched. The repulsive phases of the model are also of interest, in particular, in relation to non-Fermi-liquid behavior; they deserve further study. Finally, we would like to make connection to a seemingly unrelated phenomenon. The positive semidefinite (frustration-free) Haldane-Rezayi Hamiltonian [38,39] $\quad H=\sum_{0<j<L} H_{j}, \quad H_{j}=\sum_{k, k^{\prime}} \eta_{k} \eta_{k^{\prime}} \vec{T}_{k}^{+} \cdot \vec{T}_{k^{\prime}}^{-} \quad$ with $T_{0, k}^{+}=\left(c_{j+k \uparrow}^{\dagger} c_{j-k \downarrow}^{\dagger}+c_{j+k \downarrow}^{\dagger} c_{j-k \uparrow}^{\dagger}\right) / \sqrt{2}, \quad T_{1, k}^{+}=c_{j+k \uparrow}^{\dagger} c_{j-k \uparrow}^{\dagger}$, $T_{-1, k}^{+}=c_{j+k \downarrow}^{\dagger} c_{j-k \downarrow}^{\dagger}$, defined in a cylinder $\left(k, k^{\prime} \in[-j, j]\right.$ are angular momenta indexes), stabilizes a gapless zero mode at filling fraction $v=1 / 2$ representing a non-Abelian fractional quantum Hall trial state [40]. We have shown that (positive semidefinite) Hamiltonian $H_{j}$ is an element of $\mathrm{SO}(5)$ but, as a corollary of this work, it is not integrable à la RG. As it is a (repulsive) pairing-only Hamiltonian, it is expected that quartet correlations become relevant. Interestingly, each $H_{j}$ has a macroscopically degenerate zero-energy subspace and the intersection of their kernels results in the Haldane-Rezayi state.

Acknowledgments. We acknowledge illuminating discussions with H. Godfrin and E. Thuneberg on the nature of phase transitions in superfluid ${ }^{3} \mathrm{He}$ subject to strong magnetic fields, and G, Volovik for pointing out Ref. [14]. The open-source Python package QuSpin [41] was used for exact diagonalization. S.L.-H. acknowledges financial support from Mexican CONACyT Project No. CB2015-01/255702. J.D. is supported by the Spanish Ministerio de Ciencia e Innovación, and the European regional development fund (FEDER) under Project No. PGC2018-094180-B-I00. S.L.-H. and J.D. acknowledge financial support from Spanish Collaboration Grant No. I-COOP2017 Ref:COOPB20289. G.O. and W.H. acknowledge support from US Department of Energy Grant No. DE-SC0020343.
[1] B. Sutherland, Beautiful Models: 70 Years of Exactly Solved Quantum Many-Body Problems (World Scientific, 2004).

[2] M. Gaudin, Modèles Exactement Résolus (EDP Sciences, 1995).

[3] G. Ortiz, R. Somma, J. Dukelsky, and S. Rombouts, Exactlysolvable models derived from a generalized Gaudin algebra, Nucl. Phys. B 707, 421 (2005).
[4] G. E. Volovik, The Universe in a Helium Droplet (Oxford University Press, 2009).

[5] A. de Visser, Magnetic field-boosted superconductivity, Phys. Today 73(11), 44 (2020).

[6] V. Galitski, I. Spielman, and G. Juzeliūnas, Artificial gauge fields with ultracold atoms, Phys. Today 72(1), 38 (2019). 
[7] K. T. Hecht, Five-dimensional quasispin. Exact solutions of a pairing Hamiltonian in the $J-T$ scheme, Phys. Rev. 139, B794 (1965).

[8] J. Ginocchio, Generalized quasi-spin in neutron-proton systems, Nucl. Phys. 74, 321 (1965).

[9] J. Dukelsky, C. Esebbag, and P. Schuck, Class of Exactly Solvable Pairing Models, Phys. Rev. Lett. 87, 066403 (2001).

[10] J. Dukelsky, S. Pittel, and G. Sierra, Colloquium: Exactly solvable Richardson-Gaudin models for many-body quantum systems, Rev. Mod. Phys. 76, 643 (2004).

[11] J. Links, H.-Q. Zhou, M. D. Gould, and R. H. McKenzie, Integrability and exact spectrum of a pairing model for nucleons, J. Phys. A: Math. Gen. 35, 6459 (2002).

[12] J. Dukelsky, V. G. Gueorguiev, P. Van Isacker, S. Dimitrova, B. Errea, and S. Lerma H., Exact Solution of the Isovector Neutron-Proton Pairing Hamiltonian, Phys. Rev. Lett. 96, 072503 (2006).

[13] A. J. Leggett, A theoretical description of the new phases of liquid ${ }^{3} \mathrm{He}$, Rev. Mod. Phys. 47, 331 (1975).

[14] Y. Hasegawa, T. Usagawa, and F. Iwamoto, Application of the 5 -dimensional spin to the theory of superfluid ${ }^{3} \mathrm{He}$, Prog. Theor. Phys. 62, 1458 (1979).

[15] H.-B. Zhang, M.-L. Ge, and K. Xue, SO(5) structure of $p$-wave superconductivity for the spin-dipole interaction model, J. Phys. A: Math. Gen. 35, L7 (2002).

[16] S. Murakami, N. Nagaosa, and M. Sigrist, SO(5) Model of $p$ Wave Superconductivity and Ferromagnetism, Phys. Rev. Lett. 82, 2939 (1999).

[17] C. Wu, J.-P. Hu, and S.-C. Zhang, Exact SO(5) Symmetry in the Spin-3/2 Fermionic System, Phys. Rev. Lett. 91, 186402 (2003).

[18] E. Demler, W. Hanke, and S.-C. Zhang, SO(5) theory of antiferromagnetism and superconductivity, Rev. Mod. Phys. 76, 909 (2004).

[19] C. Wu, Competing Orders in One-Dimensional Spin-3/2 Fermionic Systems, Phys. Rev. Lett. 95, 266404 (2005).

[20] See Supplemental Material at http://link.aps.org/supplemental/ 10.1103/PhysRevB.104.L060503 for fermionic representation and commutators of the $\mathrm{SO}(5)$ algebra, derivation of the integrable Hamiltonian and coherent-state mean-field along with energy scaling in the thermodynamic limit, quartet correlations arising from $\mathrm{SO}(5)$ pairing, a discussion of the highly degenerate flat-band limits present in fermionic pairing models including a modification of the $\mathrm{SO}(5)$ Hamiltonian, and an animation of the spectral parameters as a function of coupling.

[21] Y. Hasegawa, Superfluid ${ }^{3} \mathrm{He}$ in a magnetic field, Prog. Theor. Phys. 63, 1040 (1980).

[22] S. Sachdev and J. Ye, Gapless Spin-Fluid Ground State in a Random Quantum Heisenberg Magnet, Phys. Rev. Lett. 70, 3339 (1993).

[23] S. Lerma-H and B. Errea, SU(3) Richardson-Gaudin models: Three-level systems, J. Phys. A: Math. Theor. 40, 4125 (2007).
[24] B. Errea, J. Dukelsky, and G. Ortiz, Breached pairing in trapped three-color atomic Fermi gases, Phys. Rev. A 79, 051603(R) (2009).

[25] B. Errea, Generalización de modelos de Richardson-Gaudin a álgebras de rango dos y su aplicación en física nuclear, Ph.D. thesis, Universidad Autónoma de Madrid, Departamento de Física Teórica, 2009.

[26] R. W. Richardson, New class of solvable and integrable manybody models, arXiv:cond-mat/0203512.

[27] S. M. A. Rombouts, J. Dukelsky, and G. Ortiz, Quantum phase diagram of the integrable $p_{x}+i p_{y}$ fermionic superfluid, Phys. Rev. B 82, 224510 (2010).

[28] G. Ortiz, J. Dukelsky, E. Cobanera, C. Esebbag, and C. Beenakker, Many-Body Characterization of ParticleConserving Topological Superfluids, Phys. Rev. Lett. 113, 267002 (2014).

[29] G. Ortiz and E. Cobanera, What is a particle-conserving topological superfluid? The fate of Majorana modes beyond mean-field theory, Ann. Phys. 372, 357 (2016).

[30] D. J. Rowe, M. J. Carvalho, and J. Repka, Dual pairing of symmetry and dynamical groups in physics, Rev. Mod. Phys. 84, 711 (2012).

[31] A. Tohsaki, H. Horiuchi, P. Schuck, and G. Röpke, Alpha Cluster Condensation in ${ }^{12} \mathrm{C}$ and ${ }^{16} \mathrm{O}$, Phys. Rev. Lett. 87, 192501 (2001).

[32] N. Sandulescu, D. Negrea, J. Dukelsky, and C. W. Johnson, Quartet condensation and isovector pairing correlations in $n=z$ nuclei, Phys. Rev. C 85, 061303(R) (2012).

[33] D. Negrea and N. Sandulescu, Isovector proton-neutron pairing and Wigner energy in Hartree-Fock mean-field calculations, Phys. Rev. C 90, 024322 (2014).

[34] D. M. Lee, The extraordinary phases of liquid ${ }^{3} \mathrm{He}$, Rev. Mod. Phys. 69, 645 (1997).

[35] A. Perelomov, Generalized Coherent States and Their Applications (Springer, 1986).

[36] G. Baym and C. Pethick, Landau Fermi-Liquid Theory: Concepts and Applications (Wiley-VCH, 1991).

[37] G. Ortiz, Z. Nussinov, J. Dukelsky, and A. Seidel, Repulsive interactions in quantum Hall systems as a pairing problem, Phys. Rev. B 88, 165303 (2013).

[38] F. D. M. Haldane and E. H. Rezayi, Spin-Singlet Wave Function for the Half-Integral Quantum Hall Effect, Phys. Rev. Lett. 60, 956 (1988).

[39] A. Weerasinghe and A. Seidel, Thin torus perturbative analysis of elementary excitations in the Gaffnian and Haldane-Rezayi quantum Hall states, Phys. Rev. B 90, 125146 (2014).

[40] A. Seidel and K. Yang, Gapless excitations in the HaldaneRezayi state: The thin-torus limit, Phys. Rev. B 84, 085122 (2011).

[41] P. Weinberg and M. Bukov, QuSpin: A Python Package for Dynamics and Exact Diagonalisation of Quantum Many Body Systems, Part I: Spin chains, SciPost Phys. 2, 003 (2017). 